\title{
Robot Companion: A Social-Force based approach with Human Awareness-Navigation in Crowded Environments
}

\author{
Gonzalo Ferrer*, Anaís Garrell* and Alberto Sanfeliu \\ *indicates equal contributions
}

\begin{abstract}
Robots accompanying humans is one of the core capacities every service robot deployed in urban settings should have. We present a novel robot companion approach based on the so-called Social Force Model (SFM). A new model of robotperson interaction is obtained using the SFM which is suited for our robots Tibi and Dabo. Additionally, we propose an interactive scheme for robot's human-awareness navigation using the SFM and prediction information. Moreover, we present a new metric to evaluate the robot companion performance based on vital spaces and comfortableness criteria. Also, a multimodal human feedback is proposed to enhance the behavior of the system. The validation of the model is accomplished throughout an extensive set of simulations and real-life experiments.
\end{abstract}

\section{INTRODUCTION}

Nowadays, robots interact naturally with people and their environment. Thus, urban robots require some tools in order to successfully serve their purpose of being useful to people. The robot companion is a basic tool every urban robot should have, and it responds the basic necessity of accompany people in a safety and natural way, see Fig. 1.

In recent years, an increasing area of interest is the development of autonomous companion robots [1]. Researchers are making efforts on performing human-robot interaction in a more natural way. A robot companion should detect the human operator and conduct his/her commands [2].

Research into human-robot interaction in the field of companion robots is still new in comparison to traditional service robotics, such as robots serving food in hospitals or providing specific security services. Therefore, prior research in this particular field is relatively minimal [3]. Most of the current research predominantly studies robots that participate in social-human interactions as companions [4]. Further research shown that there are other mediating factors, which can impact this preference, such as a persons experience with robots [5], gender [6] or in which part of the room she was standing or sitting [7].

Robot companion is a multidisciplinary field of robotics in which intervenes a mixture of subjects such as perception, robot navigation and human robot interaction. Despite the heterogeneity of the subjects treated, the problem can not be tackled independently but in a holistic way, which is not an easy endeavor.

Work supported by the Spanish Ministry of Science and Innovation under project RobTaskCoop(DPI2010-17112).

The authors are with the Institut de Robòtica i Informàtica Industrial (CSIC-UPC). Llorens Artigas 4-6, 08028 Barcelona, Spain. \{gferrer,agarrell, sanfeliu\}@iri.upc.edu

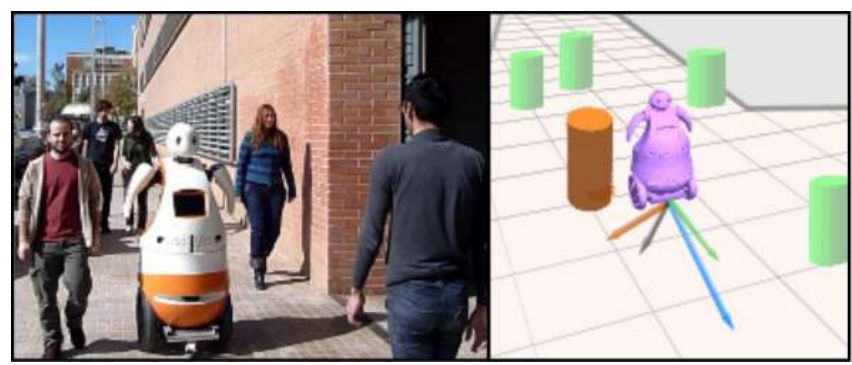

Fig. 1. Tibi accompanies a person. Left: Person being accompanied by Tibi in an urban area. Right: The same scene using the system interface.

In the present paper, we use the Social Force Model (SFM) introduced by Helbing [8] to model the social interactions, more concretely we obtain a robot-person interaction force parameters specifically suited for Tibi robot [9]. To the best of the authors' knowledge, no other work describes robotperson interactions using the SFM.

We go deeper into the development of the SFM for robot interactions. This work presents a powerful scheme for robot's human-awareness navigation based on the socialforces concept. A social aware navigation is well suited for a robot companion task. To this end, additional considerations are required to make the system work properly, such as prediction information and a learning stage.

Moreover, we introduce a new metric to evaluate in general the robot companion performance, based on vital spaces and comfortableness criteria. Since the verification of man-in-theloop systems is fuzzy, we require an analytical metric that justifies the behavior of our robot companion approach.

In addition, we present a model of human feedback response of the behavior of the system. Given the uncertainty associated to this problem, we believe that the interaction system can enhance the accuracy of the robot companion approach: the interaction can be achieved by showing the robot a better companion behavior, while simultaneously, the human can feedback the system to improve the robot performance. The validation of the model is accomplished throughout an extensive set of simulations and real-life experiments.

In the remainder of the paper we start by introducing the theory of the social force model. Section III briefly describes the human motion predictor. Section IV presents robot's human-aware navigation and a novel metric to evaluate the 
performance to accompany a person. Results and conclusions are presented in sections $\mathrm{V}$ and VI, respectively.

\section{Social-Force Model}

In order to achieve a model capable of represent the interactions between a pedestrian and a robot, we were inspired by works of Helbing [8] and Zanlungo [10]. Their main contribution is the following idea: changes in behavior (trajectory) can be explained in terms of social fields or forces. However, the cited works do not consider the interaction between a person and a robot, which is one of the contributions of the present work.

Formally, the social forces model assumes that a pedestrian $p_{i}$ with mass $m_{i}$ tries to move at a certain desired speed $v_{i}^{0}$ in a desired direction $\boldsymbol{e}_{i}$, i.e., with desired velocity $\boldsymbol{v}_{i}^{0}=v_{i}^{0} \boldsymbol{e}_{i}$.

Hence, the basic equation of motion for a pedestrian is given by a social force term:

$$
\frac{d \boldsymbol{v}_{i}(t)}{d t} m_{i}=\boldsymbol{F}_{i}(t)
$$

and describes the movements of the pedestrian $p_{i}$ over time. For the sake of simplicity, we will value $m_{i}$ as the unity for all the persons considered.

A person wants to keep his/her desired velocity through the steering force, $f_{i}{ }^{\text {goal }}$, but is also influenced by other pedestrians $p_{j}, \boldsymbol{f}_{i, j}^{i n t}$, by obstacles, $\boldsymbol{f}_{i, o}^{i n t}$ and, in the present study we model the robot interaction $\boldsymbol{f}_{i, r}^{i n t}$. The resulting force $\boldsymbol{F}_{i}$ governs the trajectory described by the target $p_{i}$.

$$
\boldsymbol{F}_{i}=\boldsymbol{f}_{i}^{\text {goal }}+\boldsymbol{F}_{i}{ }^{i n t}
$$

Below, the description of each component of $\boldsymbol{F}_{i}$ is presented. Assuming that pedestrian tries to adapt his or her velocity within a relaxation time $k^{-1}, f_{i}^{\text {goal }}$ is given by:

$$
\boldsymbol{f}_{i}^{\text {goal }}=k\left(\boldsymbol{v}_{i}^{0}-\boldsymbol{v}_{i}\right)
$$

Furthermore, repulsive effects from the influences of other people, obstacles and robot in the environment are described by an interaction force $F_{i}^{i n t}$. This force prevents humans from walking along their intended direction, moreover, it is modeled as a summation of forces either introduced by people $p_{i}$, by static obstacles in the environment $o$ or the robot $r$.

$$
\boldsymbol{F}_{i}^{i n t}=\sum_{j \in P} \boldsymbol{f}_{i, j}^{i n t}+\sum_{o \in O} \boldsymbol{f}_{i, o}^{i n t}+\boldsymbol{f}_{i, r}^{i n t}
$$

where, $P$ is the set of people moving in the environment where the human interacts and $O$ is the set of obstacles. These forces are modeled as:

$$
\boldsymbol{f}_{i, q}^{i n t}=A_{q} e^{\left(d_{q}-d_{i, q}\right) / B_{q}} \frac{\boldsymbol{d}_{i, q}}{d_{i, q}}
$$

here, $q \in P \cup O \cup\{r\}$ is either a person, an object of the environment or the robot. $A_{q}$ and $B_{q}$ denote respectively the strength and range of interaction force, $d_{q}$ is the sum of the radii of a pedestrian and an entity and $\boldsymbol{d}_{i, q} \equiv \boldsymbol{r}_{i}-$ $\boldsymbol{r}_{q}$. In order to calculate the Euclidean distance between $p_{i}$ and the entity $q$, humans and objects are assumed to be of circular shape with radii $r_{i}$ and $r_{q}$. The parameters $A_{q}, B_{q}, d_{q}$ are deffined depending on the nature of the object. In this paper we obtain the parameters describing the robot-person interaction since, to the authors' knowledge, these parameters had not been obtained before.

Given the limited field of view of humans, influences might not be isotropic. This is formally expressed by scaling the interaction forces with an anisotropic factor depending on $\varphi_{p, q}$ between $\boldsymbol{v}_{i}$ and $\boldsymbol{d}_{i, q}$

$$
w\left(\varphi_{i, q}\right)=\left(\lambda+(1-\lambda) \frac{1+\cos \left(\varphi_{i, q}\right)}{2}\right)
$$

where $\lambda$ defines the strength of the anisotropic factor,

$$
\cos \left(\varphi_{i, q}\right)=-\boldsymbol{n}_{i, q} \cdot \boldsymbol{e}_{r}
$$

The term $\boldsymbol{n}_{i, q}$ is the normalized vector pointing from $\mathrm{q}$ to person $p_{i}$ which describes the direction of the force.

\section{A. Parameters Learning}

We consider three kinds of interaction forces: personperson, person-obstacle and person-robot. The first and the second interactions has been studied in previous papers like [8], [10]. However, the person-robot interaction parameters were not directly obtained in any previous work, thereby, in this section we present a learning method to obtain the parameters $\left\{A_{p r}, B_{p r}, \lambda_{p r}, d_{p r}\right\}$.

We decouple the training in two steps: firstly, we optimize the intrinsic parameters of the model forces $\{k\}$ describing the expected human trajectories under no external constrains. Secondly, we optimize the extrinsic parameters of the force interaction model $\left\{A_{p r}, B_{p r}, \lambda_{p r}, d_{p r}\right\}$ under the presence of a moving robot, making sure it is the only external force altering the outcome of the described trajectory. All optimizations used to learn the model forces parameters are carried out using genetic optimization algorithms [11] minimizing the following error function throughout all $N$ training trajectories:

$$
\{A, B, \lambda, d\}=\arg \min _{\{A, B, \lambda, d\}}\left\{\sum_{N} \sum_{\text {time }}\left\|\mathbf{x}_{\mathbf{o}}(\mathbf{t})-\mathbf{x}_{\mathbf{e}}(\mathbf{t})\right\|\right\}
$$

where $\mathbf{x}_{\mathbf{o}}$ is the person's observed position and $\mathbf{x}_{\mathbf{e}}$ is the value expected after propagating accordingly to $F_{i}$.

\section{PEOPle PREDiction}

We require a model capable of forecasting the set of trajectories that any person might describe at any time, specifically in urban settings. As we are using the social forces model proposed by Helbing [8], we require information regarding the final destination a person aims to, that is, a long-term intentionality prediction method.

In order to predict to which destination the target is aiming to, we have used a geometrical approach in which a Bayesian predictor calculates the person posteriori probabilities to reach all destinations in the scene. The problem is treated as a sequential data classification, where orientation information with respect to each destination is required to infer the most expectable goal, making use of a variant of the Sliding Window approach.

Therefore, we can obtain a motion propagation to all destinations in the scene and the probability to occur of each future trajectory. This information is useful, specially combined with the SFM, which requires destinations, that is, 
long term intentionality predictions, in order to calculate the driven forces to a final goal. For a more detailed discussion on the prediction issue, see [12].

\section{Human-Awareness NAVIGATION}

The requirements for a social navigation system considered in this paper are: a general social interaction model based on the SFM (Sec. II), a pedestrian detector system and a prediction algorithm to estimate the best suited destination a persons aims to. These independent topics are aggregated to build a unified navigation framework, using the following idea: the robot is considered as a social agent moving naturally in human environments accordingly to the SocialForce Model, and thus, aiming to a destination and reacting to obstacles and persons. Furthermore, we believe that a more humanized navigation, in the sense that the robot responds to the SFM, will highly increase the acceptance over pedestrians, due to the similarities between the robot behavior and the expected behavior of another pedestrian.

To this end, we propose a novel approach to the robot navigation issue called human-awareness navigation, understood as an instantaneous reaction to sensory information, driven by the social-forces centered at the robot. More precisely, we aim to obtain a short-term goal-driven robot navigation ruled by the SFM. In addition, we make use of the SFM framework to successfully accompany a person while safely navigating in a crowded environment, avoiding either static and dynamical objects.

Thereby, it is mandatory to clearly formulate all the social-forces (Sec. II) intervening in the human-awareness navigation approach. The following equations are straightforward derivations of the eqs. 2-6. The force to the target's destination is inferred by using the intentionality prediction, and thus the robot aims to the target's most expectable destination:

$$
\boldsymbol{f}_{r, \text { dest }}^{\text {goal }}=k_{r}\left(\boldsymbol{v}_{r}^{0}-\boldsymbol{v}_{r}\right)
$$

The forces of interaction due to pedestrians are the repulsive forces each person generates to the robot, as follows:

$$
\boldsymbol{F}_{r}^{\text {per }}=\sum_{j \in P} \boldsymbol{f}_{r, j}^{\text {int }}
$$

where the forces $f_{r, j}^{i n t}$ represent the interaction between the pedestrian $j$ and the robot:

$$
\boldsymbol{f}_{r, j}^{i n t}=A_{r p} e^{\left(d_{r p}-d_{r, j}\right) / B_{r p}} w\left(\varphi_{r, j}, \lambda_{r p}\right)
$$

which is the formulation of the spherical force (Eq. 5) using the parameters $\left\{A_{p r}, B_{p r}, \lambda_{p r}, d_{p r}\right\}$. These parameters correspond to the person-to-robot interaction, and in general are dependent of the robotic platform used.

Correspondingly, the interaction between robot and obstacles is modelled as:

$$
\boldsymbol{F}_{r}^{o b s}=\sum_{o \in O} \boldsymbol{f}_{r, o}^{i n t}
$$

where $f_{r, o}^{i n t}$ is obtained following

$$
\boldsymbol{f}_{r, o}^{\text {int }}=A_{r o} e^{\left(d_{r o}-d_{r, o}\right) / B_{r o}} w\left(\varphi_{r, o}, \lambda_{r o}\right)
$$

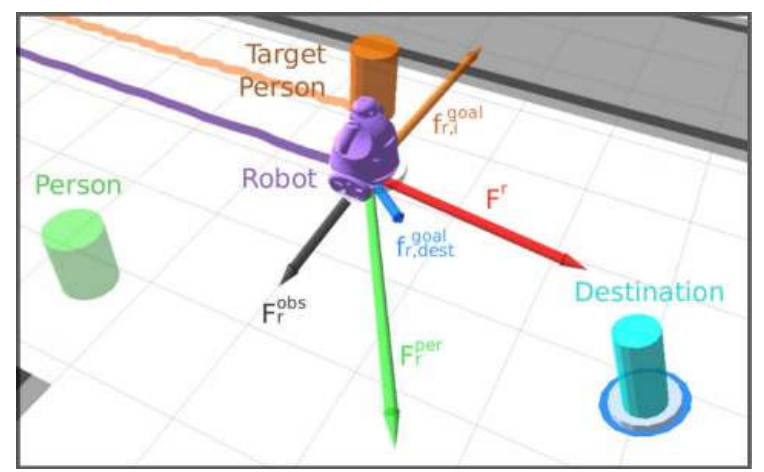

Fig. 2. Robot's Social Forces: Forces applied to the robot while accompanies a person.

using the specific parameters $\left\{A_{r o}, B_{r o}, \lambda_{r o}, d_{r o}\right\}$ corresponding to the interaction person-obstacle.

As can be seen in Fig. 2, we have defined an additional destination to the robot approach. The robot aims to the target person in order to accompany him/her, following the Eq. 9 .

Similarly as presented in section II, repulsive effects from the influences of other people and obstacles in the environment are described by an interaction force which is a sum of forces either introduced by people or by static obstacles in the environment.

In contrast to the social-force model, two different goals appear. Firstly, a force makes the robot drive towards the predicted destination $\boldsymbol{f}_{r, \text { dest }}^{\text {goal }}$. Furthermore, the robot must approach the person who accompanies, and hence a second goal pushes the robot to move closer to the person $p_{i}, \boldsymbol{f}_{r, i}^{\text {goal }}$. The trade off of these forces in addition to the interacting forces, describes the resultant force governing the robot movement:

$$
\boldsymbol{F}^{r}=\alpha \boldsymbol{f}_{r, \text { dest }}^{\text {goal }}+\beta \boldsymbol{f}_{r, i}^{\text {goal }}+\gamma \boldsymbol{F}_{r}^{\text {per }}+\delta \boldsymbol{F}_{r}^{\text {obs }}
$$

Once obtained the reactive force action, the system behaves consequently to these stimuli and propagates linearly its position and velocity according to this force value.

The most interesting part of the system so far, resides in the fact that the approach proposed does not require static targets, the robot is able to navigate near to moving persons. Moreover, it can accompany those people who aim to the same destination. The following section discusses the procedure to obtain the value of the parameters $\{\alpha, \beta, \gamma, \delta\}$ and how they are updated.

\section{A. Interactive Learning}

In order to learn the values of the introduced parameters $\{\alpha, \beta, \gamma, \delta\}$, we use an Interactive Learning scheme [13] under the shape of the person's response to the stimuli generated by the robot. This method helps to enlighten the nature of the model, in addition to generate controlled interaction forces that otherwise would be extremely complicated to generate.

The on-line feedback comes from the target person to whom the robot tries to approach. The interaction provided by a human agent by using a wii remote control has been defined. Here, we expect to receive a feedback measure of the subjective comfortableness of the target being approached. 
This feedback is a subjective measure, nevertheless, we have modeled a system weighting the contribution of all active forces. Volunteers had a wii remote control. Participants were told to press the button ' + ' if they wanted the robot to get closer to them. However, if people preferred the robot to move directly to the destination, they should push button '-'. Below, parameters' variations depending on people's feedback are presented.

Firstly, we can define the function $N(T)$ as follows:

$$
N(T)=\sum_{t=0}^{T} \epsilon(t)
$$

where $\epsilon(t)$ is expressed as:

$$
\epsilon(t)= \begin{cases}+1 & \text { if human presses button ' }+ \text { ' at time } t \\ -1 & \text { if human presses button '-' at time } t\end{cases}
$$

$N(T)$ is the difference between the number of times the person presses button ' + ' and button '-' at time $T$. Then, $N(T) \geq 0$, if $N(T)<0$ we impose $N(T)=0$.

Secondly, the forces that appear during the process of accompanying vary according to the distance between the robot and the person. Then, the variation of the parameters will change depending on such distance.

Formally, if $h(N(T))$ denotes the function corresponding to human's response, it can be expressed as:

$$
h(N(T))= \begin{cases}\alpha(N(T)), \beta(N(T)) & \text { if } d_{r, i} \geq w\left(\varphi_{r, i}\right) \\ \gamma(N(T)), \delta(N(T)) & \text { if } d_{r, i}<w\left(\varphi_{r, i}\right)\end{cases}
$$

Where, $\{\alpha(N(T)), \beta(N(T)), \gamma(N(T)), \delta(N(T))\}$ is the set of weighting functions for the parameters $\{\alpha, \beta, \gamma, \delta\}$, $d_{r, i}$ is the distance between the robot and the person, and, $w\left(\varphi_{r, i}\right)$ represents the personal space of a person, see eq. 6 .

Below, the weighting functions are presented.

Force to the target destination $\alpha$ : We infer the destination of the target by using the intentionality prediction described in section III, and thus the robot aims to the most expectable target's destination. As it has been described above, a parameter $\alpha$ controls the magnitude of the force $f_{r, d e s t}^{\text {goal }}$. The value of this parameter is computed as follows:

$$
\alpha(N(T))=\log (1+N(T) / 4)
$$

Force to the person being accompanied $\boldsymbol{\beta}$ : An attractive force towards the accompanied person has been described. Either the current target position as well the expected motion prediction are known. The parameter $\beta$ controls the magnitude of the force $\boldsymbol{f}_{r, i}^{\text {goal }}$. The value of this parameter is computed as follows:

$$
\beta(N(T))=\log (1+N(T))
$$

Force of interaction with people $\gamma$ : A repulsive force due to the relative position and velocity between the robot and people must be considered, $\sum_{j \in P} f_{r, j}^{i n t}$, this force is controlled by the parameter $\gamma$. The value of $\gamma$ is defined as:

$$
\gamma(N(T))=\ln (1+N(T) / 2)
$$

Force of interaction with obstacles $\delta$ : Finally, a repulsive force due to the relative position and velocity between the

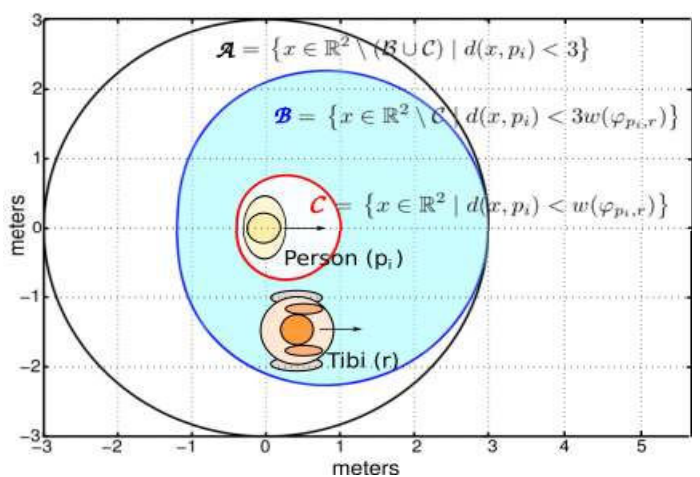

Fig. 3. Quantitative Metrics: Diagram of the areas used in the evaluation of the robot's performance.

robot and obstacles has to be considered, $\sum_{o \in O} \boldsymbol{f}_{r, o}^{i n t}$, this force is controlled by the parameter $\gamma$. The value of $\gamma$ has been computed under simulation.

The combination of these four forces determines the behavior of the robot while physically approaching a person. The feedback provided refines the weights of the force parameters and we can infer an interactive behavior where the person feels comfortable under the presence of the robot.

\section{B. Quantitative Metrics}

To evaluate the performance of the task accomplished by the robot, a quantitative metric is defined. This assessment is based on "proxemics", proposed in [14]. This work considers the following taxonomy of distances between people:

- Intimate distance: the presence of another person is unmistakable $(0-45 \mathrm{~cm})$.

- Personal distance: comfortable spacing (45cm-1.22m).

- Social distance: limited involvement (1.22m-3m).

- Public distance: outside circle of involvement ( $>3 \mathrm{~m})$.

To define the metric used in the present work, three different areas must be defined: (i) Human's vital space $\mathcal{C}$, robot's navigation has to be socially accepted by the person being accompanied, it is necessary that the robot does not perturb the human's vital space, eq. 21. (ii) Social distance area $\mathcal{A}$, robots must be allocated in an acceptance social distance. (iii) Finally, the robot should be in the human's field of view as they interact during the performance of the task $\mathcal{B}$.

$$
\begin{aligned}
\mathcal{A} & =\left\{x \in \mathbb{R}^{2} \backslash(\mathcal{B} \cup \mathcal{C}) \mid d\left(x, p_{i}\right)<3\right\} \\
\mathcal{B} & =\left\{x \in \mathbb{R}^{2} \backslash \mathcal{C} \mid d\left(x, p_{i}\right)<3 w\left(\varphi_{p_{i}, r}\right)\right\} \\
\mathcal{C} & =\left\{x \in \mathbb{R}^{2} \mid d\left(x, p_{i}\right)<w\left(\varphi_{p_{i}, r}\right)\right\}
\end{aligned}
$$

where $w\left(\varphi_{p_{i}, r}\right)$ is defined in eq. 6 .

Moreover, robot can be represented as a circle of 1 meter of diameter, with center robot's position $r, \mathcal{R}=\{x \in$ $\left.\mathbb{R}^{2} \mid d(x, r)<0.5\right\}$, whose area is $|\mathcal{R}|=\frac{\pi}{4}$.

Thus, we can now define the performance of the task accomplished by the robot, depending on human's position $p_{i}$ and robot's position $r$.

$$
\mathrm{P}\left(r, p_{i}\right)=\frac{1}{|\mathcal{R}|} \int_{\mathcal{B} \cap \mathcal{R}} d x+\frac{1}{2|\mathcal{R}|} \int_{\mathcal{A} \cap \mathcal{R}} d x \in[0,1]
$$


The function presented has the maximum performance in the area described by $\mathcal{B}$, since it is the area of human's field of view and where the interaction between the robot and the human is maximal. Additionally, the area $\mathcal{A}$, is a partial success, since this area is less tolerable by humans. Finally, in the area described further than three meters there is no interaction, and therefore its performance is zero.

\section{EXPERIMENTS}

In previous sections, we have presented the theoretical aspects of a wide variety of topics, including a social force model (SFM), a long-term predictor of motion intentionality and a human-awareness navigation. Additionally, we have discussed how these independent topics can be unified into the same robot companion framework.

\section{A. Robotic Platform, Environment and Implementation}

To conduct the experiments and to test the approach presented, we have used two twin mobile service robots, called Tibi and Dabo (Fig. 6-top), designed to work in urban pedestrian areas and interact with people [1].

The experimental areas where the experiments were conducted are the Barcelona Robot Lab (BRL), and the Facultat de Matemàtiques i Estadística (FME). Both are outdoor urban environments covering over $10.000 \mathrm{~m}^{2}$, with multiple ramps, stairs and obstacles such as bicycle stands, trashcans or flower pots.

We are using a probabilistic localization, an implementation of the adaptive (or KLD-sampling) Monte Carlo localization approach, which uses a particle filter to track the pose of a robot against a known map [15]. Our implementation of the people detector is fundamentally based on laser information ([16]). This approach uses a boosting method to classify if a group of laser points is a human being. And the final requirement is a people tracking, which implementation follows a similar approach of the work presented in [17].

\section{B. SFM parameters}

The first step required for the robot companion is the study of the SFM that governs human motion in general. We consider three kinds of interaction forces: person-person, person-obstacle and person-robot. The first and the second interactions have been studied in previous papers like [8], [10] and [18]. However, the person-robot interaction parameters were not directly obtained in any previous work, thereby, in this section we present the results obtained for the parameters $\left\{A_{p r}, B_{p r}, \lambda_{p r}, d_{p r}\right\}$.

As discussed in Sec. II-A, we have recorded two different databases of human motion in a real scenario. During the first part, we optimize the intrinsic parameter of the SFM $\{k\}$ describing the expected human trajectories under no external constrains.

The second part of the SFM parameter learning was done under the influence of the Tibi robot. We optimize the extrinsic parameters of the force interaction model $\left\{A_{r}, B_{r}, \lambda_{r}, d_{r}\right\}$ under the presence of a moving robot, making sure it is the only external force altering the outcome of trajectory described by the person.

\begin{tabular}{|l||c|c|c|c|c|}
\multicolumn{2}{|c|}{ TABLE I } \\
\hline Interaction & $\mathrm{k}$ & $\mathrm{A}$ & $\mathrm{B}$ & $\mathrm{d}$ & $\lambda$ \\
\hline \hline Per-Per [18] & 2 & 1.25 & 0.1 & 0.2 & 0.5 \\
\hline Per-Per [10] & 4.9 & 10 & 0.34 & 0.16 & 1 \\
\hline $\begin{array}{l}\text { Robot-Per } \\
\text { (our approach) }\end{array}$ & $\begin{array}{c}2.3 \\
( \pm 0.37)\end{array}$ & $\begin{array}{c}2.66 \\
( \pm 4.51)\end{array}$ & $\begin{array}{c}0.79 \\
( \pm 0.21)\end{array}$ & $\begin{array}{c}0.4 \\
( \pm 0.25)\end{array}$ & $\begin{array}{c}0.59 \\
( \pm 0.36)\end{array}$ \\
\hline
\end{tabular}

Model Forces Parameters. Parameters learned after applying

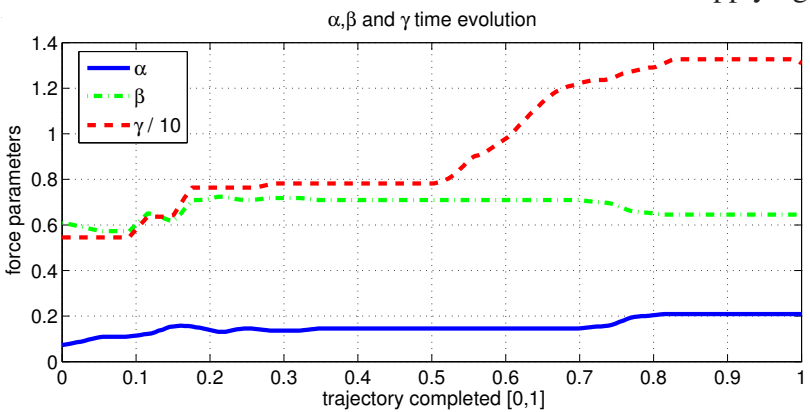

Fig. 4. Force parameters $\alpha, \beta, \gamma$ : Evolution in time from start to end of each experiment. These variables are averaged using the different results each of the participants chose during the experiment.

Table I shows the parameters learned after applying the minimization process (see Sec. II-A), using genetic algorithms, to all database trajectories. Each parameter include a standard deviation obtained after estimating each trajectory independently. In the same table, it can be seen the parameters proposed by Luber [18] and Zanlungo [10] works refered to the person-person SFM. However, in the present work, we are applying the SFM to learn the parameters for a human-robot interaction, opposite to [10], [18]. Furthermore, the standard deviation of some parameters is high, because people behave differently when they interact with robots.

\section{C. $\alpha, \beta, \gamma$ and $\delta$ parameters}

Once obtained the parameters of the SFM person-robot, we are prepared to obtain the parameters $\{\alpha, \beta, \gamma, \delta\}$. The experiment setting, using a robot in a real scenario (FME), is as follows: we explain each volunteer to naturally walk towards its chosen destination, among two options. While approaching the desired destination, the robot will accompany the volunteers and they should behave naturally.

As part of the second learning phase, the system learns the desired robot behavior as explained in Sec. IV-A. The purpose of the provided feedback is to learn a general approaching rule that defines a better robot behavior. It is provided directly by the target agent to be approached using a remote control, in this way the system automatically weights the contribution of the active forces, Sec. IV-A.

Fig. 4 shows the $\{\alpha, \beta, \gamma\}$ obtained from the user feedback that determines the robot behavior. It has been averaged using 25 different experiments and it is depicted as a function of time, normalized from the start of the experiment to its ending $t \in[0,1]$.

\section{Simulations}

In order to evaluate mathematically the correctness of the reactive navigation model, and the performance of the robot companion approach, we have built a simulated social environment. This simulated environment serves two purposes: firstly it permits a readjustment of the $\gamma$ and $\delta$ parameters, as 

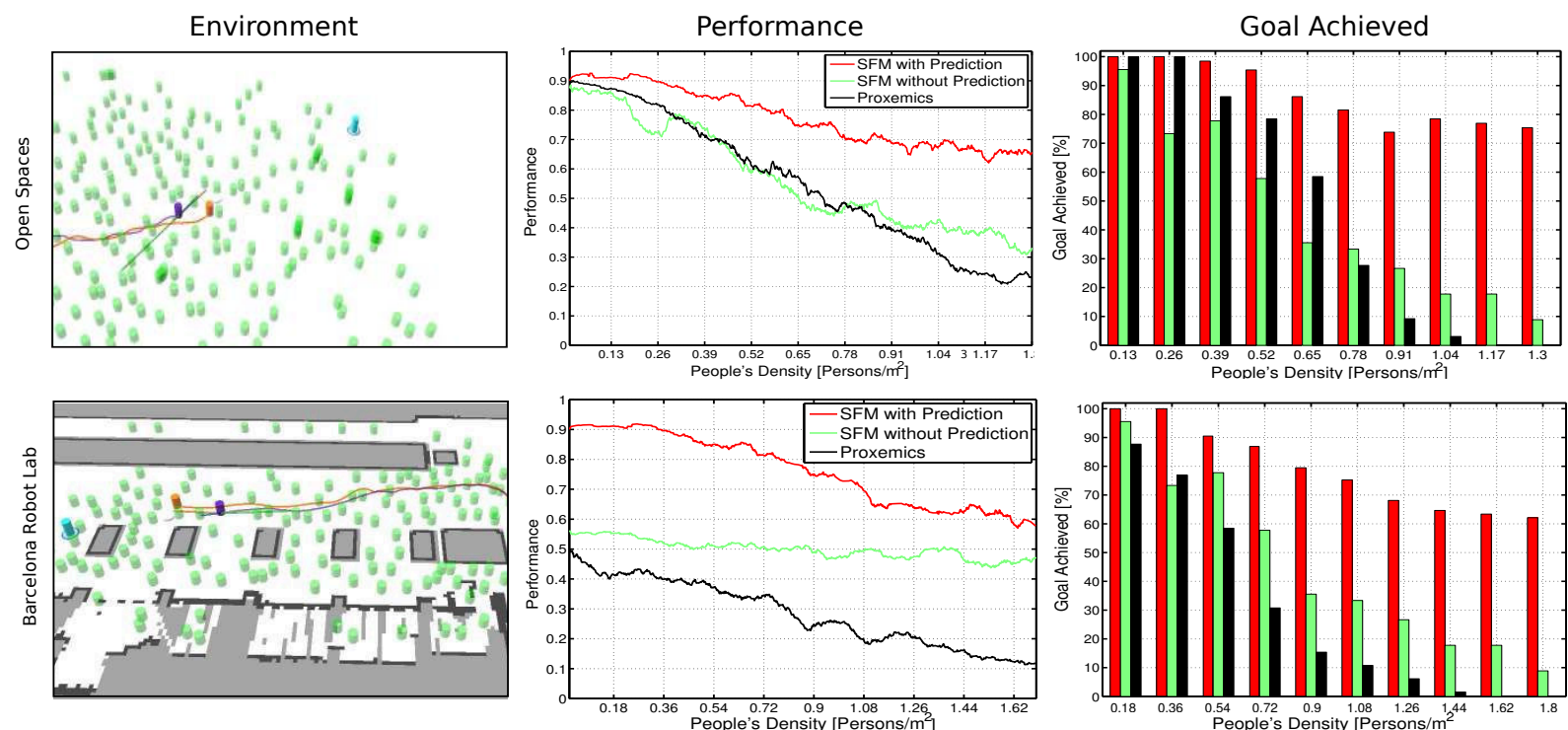

Fig. 5. Synthetic Experiments. Top: Unconstrained area. Bottom: Urban settings corresponding to the Barcelona Robot Lab. The second column corresponds to the performance presented previously; black the proxemics approach, green the SFM companion and red the SFM with prediction information. All results are function of the pedestrian density in the environment. The third column are bar diagrams showing the rate of successful robot arrivals.

the system was not tested in highly crowded environments. Secondly, the simulated environment allows us to validate the performance of the approach, using the metrics defined in Sec. IV-B, in different environments and under different density of pedestrians.

To this end, we have implemented a complete social environment, depicted in the left column in Fig. 5, which takes into account pedestrians, obstacles and robots in an interactive way and each element is reactive to its surrounding according to the SFM. By doing this, we can get a dynamical environment, in which each action of the robot alters the behavior of nearby pedestrians and vice versa.

To validate the performance of our contributions, we have prepared a set of simulations. Our method makes use of the SFM of surrounding persons and obstacles while approaching the target and additionally uses prediction information regarding the target destination to enhance its performance (red in figure). A second configuration takes into account only the SFM model (green in figure). For this reason, the avoidance of moving targets and obstacles is executed dynamically using the interaction forces in addition to the goal force. Our method is compared to a robot companion based on proxemics where the robot follows the target person, not considering the force of interactions of other persons. When some person enter the robot inner safety zone, the robot stops until the path is clear (black lines in Fig. 5).

The experiment settings have been tested in two different scenarios, as can be seen in the left column in Fig. 5, the first setting is an unconstrained area, free of obstacles, where four destinations are defined. The second is a urban settings, in which obstacles are present as well as pedestrians.

For each environment, the algorithms have been tested depending on the density of persons in the unoccupied area. To give statistical consistency to the results, more than $50 \mathrm{k}$ experiments have been carried out, only varying the initial conditions, which is the initial position of each pedestrian in the scene and the destination they are aiming to. This conditions are calculated randomly and the robot has to accompany a person under this uncertain environment. We would like to stress on the fact that the environment has a high density of persons and each person aims to a random destination. This generates rapidly a chaotic and challenging environment for the robot companion testing (see video at the project web).

Under this circumstances, we can test the stability of the method, that is, if the robot can reach the goal independently of the initial conditions and the environment condition, such as the deployment of external agents or obstacles. We have observed that most of the times, the robot or person escapes local minima thanks to the surrounding interactions and the constant steering force towards a destination.

The second column of Fig. 5 shows the overall performance of the different methods with respect to the density of pedestrians in the scene. As expected, using social interaction forces highly increases the performance, it is natural to suppose that a more awareness robot navigation would help to improve its efficiency. The predictive behavior clearly enhances the performance of the task, either in the unconstrained scenario or in the urban environment.

The third column of Fig. 5 shows an average percentage of successful arrivals to the destinations, that is, if the robot is within the companion zone (Sec. IV-B) at the moment the target achieves its destination.

\section{E. Real experiments}

Nowadays, real experimentation is mandatory in order to evaluate a robot model, independently on how many simulations have been carried out. The proposed robot companion approach, has been tested in the FME and in the Barcelona Robot Lab.

We carried out 60 experiments with different volunteers. The robot was able to achieve its goal (the target's goal) 




Fig. 6. Real-life experiments: Some examples of the conducted real experiments. Top: Dabo accompanying a person to a desired goal. Bottom: The same scene using the system interface.
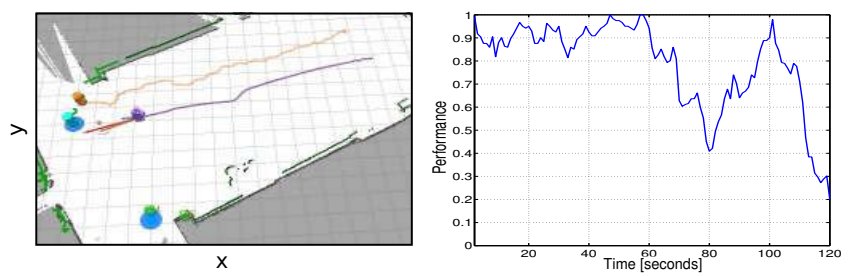

Fig. 7. Trajectories and Performance: Left: Trajectories of the robot and the volunteer. Right: Performance obtained during the experiment

in all conducted experiments. The volunteers were told to naturally walk and the robot accompanied the target using the human-awareness navigation described in Sec. IV. During the validation of the model in real experiments, we set unexpected obstacles and pedestrians in the targets path, and the robot avoided them successfully.

The performance of a robot companion experiment, and the trajectories of the robot and the volunteers for that experiment are shown in Fig. 7.

We would like to point the reader to check all the videos of synthetic and real experiments on following link http://www. iri.upc.edu/groups/Irobots/robot_companion/iros2013.php

\section{CONCLUSIONS AND FUture WORK}

We have presented a novel robot companion approach based on the so called Social-Forces Model. The major contributions of this paper are threefold. First, we obtain the force parameters of robot-person interaction, specifically suited for Tibi. We have gone one step ahead into the development of the SFM for robot interactions, we presented a powerful scheme for robot's human-awareness navigation based on the social-forces concept. A social aware navigation is well suited for a robot companion task, a better performance has been demonstrated if human interactions are taken into account and intentionality prediction information is used, specially in open spaces.

Second, the metric is also a contribution of the paper, since the verification of any system in which a human intervenes is hard to evaluate, and thus, we require an analytical metric that justifies the behavior of our robot companion approach.

Finally, we have introduced a model of human feedback that is able to obtain the set of weighting parameters for the robot companion behavior. We believe that human feedback for parameter learning is a key point for the development of robots whose purpose is interacting with people. The validation of the model has been demonstrated throughout an extensive set of simulations and real-life experiments in a urban area.

In future work, we aim to obtain more sophisticated robot behavior, by exploring the enhancement of the model of the human motion prediction.

\section{REFERENCES}

[1] A. Garrell and A. Sanfeliu, "Cooperative social robots to accompany groups of people," The International Journal of Robotics Research, vol. 31, no. 13, pp. 1675-1701, 2012.

[2] A. Haasch, S. Hohenner, S. Hüwel, M. Kleinehagenbrock, S. Lang, I. Toptsis, G. Fink, J. Fritsch, B. Wrede, and G. Sagerer, "Biron-the bielefeld robot companion," in Proc. Int. Workshop on Advances in Service Robotics, 2004.

[3] D. Wilkes, R. Pack, A. Alford, and K. Kawamura, "Hudl, a design philosophy for socially intelligent service robots," in American Association for Artificial Intelligence, 1997.

[4] H. Ishiguro, T. Ono, M. Imai, T. Maeda, T. Kanda, and R. Nakatsu, "Robovie: an interactive humanoid robot," Industrial robot: An international journal, 2001.

[5] K. Koay, E. Sisbot, D. Syrdal, M. Walters, K. Dautenhahn, and R. Alami, "Exploratory studies of a robot approaching a person in the context of handing over an object," in Proc. AAAI-Spring Symp., 2007.

[6] K. Dautenhahn, M. Walters, S. Woods, K. Koay, C. Nehaniv, A. Sisbot, R. Alami, and T. Siméon, "How may i serve you?: a robot companion approaching a seated person in a helping context," in Proc. of ACM SIGCHI/SIGART conf. on HRI, 2006.

[7] M. Walters, K. Dautenhahn, S. Woods, and K. Koay, "Robotic etiquette: results from user studies involving a fetch and carry task," in Proc. of the ACM/IEEE Int. Conf. on HRI, 2007.

[8] D. Helbing and P. Molnár, "Social force model for pedestrian dynamics," in Physical review. E, Statistical physics, plasmas, fluids, and related interdisciplinary topics, 1995.

[9] A. Sanfeliu and J. Andrade-cetto, "Ubiquitous Networking Robotics in Urban Settings," in IEEE/RSJ IROS Workshop on Network Robot Systems, 2006.

[10] F. Zanlungo, T. Ikeda, and T. Kanda, "Social force model with explicit collision prediction," EPL (Europhysics Letters), 2011.

[11] D. Goldberg, Genetic Algorithms in Search, Optimization \& Machine Learning. Addison-Wesley, 1988.

[12] G. Ferrer and A. Sanfeliu, "Bayesian human motion intentionality prediction in urban environments," Pattern Recognition Letters Journal, submitted.

[13] D. Katagami and S. Yamada, "Active teaching for an interactive learning robot," in IEEE ROMAN, 2003, pp. 181-186.

[14] E. T. Hall, The hidden dimension. Anchor Books New York, 1969.

[15] S. Thrun, W. Burgard, D. Fox, et al., Probabilistic robotics. MIT press Cambridge, MA, 2005, vol. 1.

[16] K. Arras, O. Mozos, and W. Burgard, "Using boosted features for the detection of people in 2d range data," in IEEE ICRA, 2007.

[17] M. Luber, G. Tipaldi, and K. Arras, "Place-dependent people tracking," IJRR, 2011

[18] M. Luber, J. Stork, G. Tipaldi, and K. Arras, "People tracking with human motion predictions from social forces," in IEEE ICRA, 2010. 